\title{
The Influence of Social Support on College Students' Mobile Phone Dependence: The Mediating Role of Security
}

\author{
Fu Guifang ${ }^{1}$, Shi Kexin ${ }^{1} \&$ Ye Kai ${ }^{2}$ \\ ${ }^{1}$ Department of Applied Psychology, School of Public Administration, Guangdong University of Foreign Studies, \\ Canton, China \\ ${ }^{2}$ Students' Affairs Division, Guangdong University of Foreign Studies, Canton, China \\ Correspondence: Ye Kai, Students' Affairs Division, Guangdong University of Foreign Studies, Canton, China. \\ E-mail:200411249@oamail.gdufs.edu.cn
}

Received: January 8, 2020 Accepted: February 8, 2020 Online Published: February 15, 2020

doi:10.5539/nct.v5n1p44 URL: https://doi.org/10.5539/nct.v5n1p44

This work was supported by The National Social Science Fund of China [grant number 16BSH094].

\begin{abstract}
In order to further study the current situation and relationship of college students' mobile phone dependence, social support and sense of security, a questionnaire survey was performed on 409 college students using the college students' mobile phone addiction tendency scale, social support assessment scale and sense of safety scale. The results showed that: (1) $40.24 \%$ of college students are moderate of mobile phone dependence, and $5.75 \%$ of college students had serious problem of mobile phone dependence; the social support and sense of security of college students were at the upper-middle level; there is no gender difference, no one child difference and no love difference of mobile phone dependence in college students; there is grade difference of mood change; (2) The social support and sense of security of people with high phone dependence are significantly lower than those with low phones Dependence; (3) Social dependence and sense of security have significant negative correlations and negative predictive effects on mobile phone dependence; (4) Security sense has a full mediating role between social support and mobile phone dependence, thus enhancing social support and the sense of security of college students can effectively improve their mobile phone dependence.
\end{abstract}

Keywords: social support, security, mobile phone dependence, college students

\section{Introduction}

Mobile phone dependence (MPA), also called mobile phone addiction, or pathological mobile phone use. Specifically, it means that when a person overuses a mobile phone and cannot use the mobile phone for a period of time, it will have corresponding emotional or behavioral performance.

With the widespread popularity of smart phones, mobile phones have become an indispensable tool for people's daily communication. According to data from the Statistical Bulletin of the Communications Operations Industry in 2018, the mobile phone user penetration rate has reached 112.2 units / 100 people, of which 24 provinces and cities have a mobile phone penetration rate of more than 100/100 people, and college students are the mainstream population for mobile phone use. While enjoying the convenience of mobile phones, they are also increasingly obsessed with the use of mobile phones. The problem of mobile phone dependence is becoming more and more serious.

At present some scholars have two opinions of mobile phone dependence. One is that mobile phone dependence is a normal social phenomenon, and the other is that mobile phone dependence is a behavioral addiction. People with mobile phone dependence have psychological problems (Zhao, 2016; Wang, 2016). For the many people, the most obvious manifestation is that they will always check their mobile phones before going to bed or eating even walking. When they do not bring the mobile phones or the power of the mobile phones is insufficient, they will experience panic and anxiety. Due to the freedom of time, mobile phone dependence of college students is even heavily. Mobile phone dependence not only affects the normal life of students, but also affects their physical and mental health. So it is necessary to study the problem of college students' mobile phone dependence. 
Mobile phone dependence behavior is related to many factors, such as personality traits, mental health, social support, social adaptability, self-control and loneliness and so on. Studies had found there are a significant negative correlation between mobile phone dependence on social support (Jin Rong, Wen, \& Jiang, 2015) and a significant positive correlation with loneliness (Wang, 2016). Empirical research also found that social support can also negatively predict the level of mobile phone dependence (Wei, 2012), while the total score of college students' mobile phone dependence is significantly negatively correlated with social support (Liu \& Cai, 2015). Some researches suggested college students' sense of security is at a moderately high level and social support and sense of are significantly positively correlated (J. Li, 2013). Research also found in-family support and out-of-family support, interpersonal security and negative life events were a significantly negatively correlated (Guo, P. Zhang Ping, \& Y. Zhang, 2014).

Based on the existing research, this article attempts to explore the current situation and relationship of college students' mobile phone dependence, security and social support. The results of this study could provide theoretical and practical basis for the intervention of mobile phone dependence of college students

\section{Methods}

\subsection{Participants}

Taking college students as the research object, the data was collected using online questionnaires. A total of 409 questionnaires were recovered, of which 400 were valid, with a recovery rate of $97.80 \%$. Among them, 133 are males, accounting for $33.25 \%$, others are females and the majority of the samples are females; 73 were freshmen, $18.25 \%$, and 57 are sophomores, $14.25 \%, 69$ were juniors, $17.25 \%, 182$ were juniors, $45.5 \%$, 19 were graduates and above, accounting for $4.75 \%$, and the data indicated that among the samples were mainly seniors; in the sample surveyed, 111 were in a relationship, $27.75 \%$, and 289 were not in a relationship, accounting for $72.25 \%$. The majority of the participants in the survey were single people.

\subsection{Instruments}

\subsubsection{Mobile Addiction Tendency Scale}

This study used the "Mobile Phone Addiction Tendency Scale for College Students" compiled by Xiong et al (2012). The scale has 16 items and is divided into four factors and they are withdrawal symptoms, highlighting behaviors, social comfort and mood change. Withdrawal symptoms mean that psychological and physical discomfort when the mobile phone is unavailable for a period of time. Highlighting behaviors is the use of mobile phones as the core of behavioral activities and thinking. Social comfort played a major role in the process of communication and mood change means emotional changes due to the use of mobile phones et al (2012). In this study, the Cronbach $\alpha$ coefficient of the total scale is 0.83 , the retest reliability coefficient is 0.91 , and the retest reliability of the four factors is between 0.75-0.85. In addition, this scale uses Likert's 5-point scoring method. 1-5 points represent very non-conforming, relatively non-conforming, general, relatively conforming, and very conforming. A higher total score indicates that the individual is more inclined to mobile phone addiction (Liu, Cai, 2015).

\subsubsection{Social Support Scale}

This study used the Social Support Scale designed by Xiao (1994) for the social support of college students. The scale consists of 10 items and is divided into three factors, objective support, subjective support, and utilization of social support. The retest reliability coefficient of the total scale of the scale is 0.92 , and the retest reliability coefficient interval of the three dimensions is $0.89-0.94$. The scale has good retest reliability. In this study, according to the characteristics of the test group, the "colleagues" in the fourth question in the scale was changed to "classmates", the "couples" in the fifth question was changed to "lovers", and the "children" was removed. The higher the overall score, the more social support is obtained (Xiao, 1994).

\subsubsection{Security Scale}

The study used the security questionnaire (SQ) compiled by Cong and An in 2004 for the sense of security of college students. This scale was based on Maslow's Safety-The Insecurity Scale was compiled. The scores of the safety scale and factors of normal people and neurosis patients have significant differences, indicating that the scale has good empirical validity for distinguishing normal people from neurosis patients (Liu, 2014; Guo et al., 2014). The scale's homogeneity reliability and retest reliability are 0.857 and 0.870 , respectively, which proves that the scale has better reliability. The scoring method of this table is also a five-level scoring. A is very consistent, $\mathrm{B}$ is basically consistent, $\mathrm{C}$ is neutral or uncertain, $\mathrm{D}$ is not very consistent, $\mathrm{E}$ is very non-compliant. 1-5 points are scored respectively. A higher score indicates a greater sense of security (Cong, 2004). 


\section{Results}

\subsection{Current Situation of College Students' Mobile Phone Dependence, Social Support and Sense of Security}

This study found the total average score of college students' mobile phone dependence was 46.25 , with the lowest score being 16 and the highest score being 76. According to Likert's 5-point scoring method, the average value of the scale is 48 , and the total score of university students' mobile phone dependence is very close to the average. Among them 31 people have scores between 0 and 31 , indicating that $7.75 \%$ of college students have no mobile phone dependence. There are 185 people between 32-47 points who have a slight tendency to of mobile phones dependenc, accounting for $46.25 \%$; 161 have a score between $48-63$ points, which indicates that $40.24 \%$ of college students have moderate mobile phone dependence. And there are 23 people with scores between $64-80$, indicating that $5.75 \%$ of college students have serious mobile phone dependence. Data show that college students' mobile phone dependence is more serious.

Difference analysis was used to examine the differences in mobile phone dependence of demographic variables. The results showed that: (1) there is no gender difference, one child difference and love difference of mobile phone dependence in college students; (2) there is grade difference of mood change factor.

Table 1. Descriptive statistics $(\mathrm{M} \pm \mathrm{SD})$ and difference analyses

\begin{tabular}{lllllll}
\hline Variable & & $\begin{array}{l}\text { Withdrawal } \\
\text { symptoms }\end{array}$ & $\begin{array}{l}\text { Highlighting } \\
\text { behavior }\end{array}$ & $\begin{array}{l}\text { Social } \\
\text { comfort }\end{array}$ & $\begin{array}{l}\text { Mood } \\
\text { change }\end{array}$ & Total MPA \\
\hline \multirow{2}{*}{ Sex } & Male & $18.45 \pm 4.67$ & $10.42 \pm 3.23$ & $7.71 \pm 2.65$ & $7.87 \pm 2.39$ & $44.45 \pm 10.56$ \\
& Female & $19.45 \pm 4.28$ & $10.88 \pm 3.26$ & $8.57 \pm 2.56$ & $8.28 \pm 2.32$ & $47.18 \pm 10.34$ \\
\hline $\mathrm{t}$ & & 0.03 & 0.18 & 0.002 & 0.10 & 0.02 \\
\hline \multirow{2}{*}{ Grade } & Freshman & $19.08 \pm 4.34$ & $9.92 \pm 3.01$ & $8.51 \pm 2.72$ & $8.49 \pm 2.29$ & $46.00 \pm 9.84$ \\
& Sophomore & $19.37 \pm 4.23$ & $10.67 \pm 3.39$ & $8.37 \pm 2.69$ & $7.91 \pm 2.47$ & $46.32 \pm 10.48$ \\
& Junior & $19.87 \pm 4.37$ & $11.23 \pm 3.06$ & $8.71 \pm 2.53$ & $8.87 \pm 2.21$ & $48.68 \pm 10.50$ \\
& Senior & $18.77 \pm 4.56$ & $10.88 \pm 3.25$ & $8.04 \pm 2.61$ & $7.92 \pm 2.33$ & $45.62 \pm 10.67$ \\
\hline $\mathrm{F}$ & Graduate and above & $18.89 \pm 4.22$ & $10.79 \pm 4.12$ & $7.63 \pm 2.34$ & $6.95 \pm 2.09$ & $44.26 \pm 10.53$ \\
\hline \multirow{2}{*}{ Single or not } & Yes & 0.89 & 1.67 & 1.28 & $3.96 *$ & 1.28 \\
\hline $\mathrm{t}$ & No & $19.29 \pm 4.40$ & $10.95 \pm 3.22$ & $7.90 \pm 2.51$ & $8.23 \pm 2.16$ & $46.38 \pm 10.18$ \\
\hline$* \mathrm{P}<0.05 * * \mathrm{P}<0.01 * * * \mathrm{P}<0.001$ & $19.04 \pm 4.44$ & $10.65 \pm 3.26$ & $8.41 \pm 2.65$ & $8.10 \pm 2.42$ & $46.20 \pm 10.60$ \\
\hline
\end{tabular}

$* \mathrm{P}<0.05, * * \mathrm{P}<0.01, * * * \mathrm{P}<0.001$.

\subsection{Differences of Mobile Phone Dependence Level and Social Support and Sense of Security}

According to the statistical principle of high and low grouping, the first $27 \%$ and the last $27 \%$ of the total mobile phone dependence score were divided into low and high groupings. The independent sample $t$ test was used to compare the high and low mobile phone dependence levels with Social support, sense of security. The results showed: (1) There were significant differences in social support and its sub dimension subjective support between the high and low mobile phone dependence groups. The social support and its sub dimension subjective support of college students with high mobile phone dependence were significantly lower. (2) There were significant differences between the high and low cell phone dependence groups in the sense of security and its sub dimensions of interpersonal security and control. 
Table 2. Comparative analysis of social support and sense of security between high and low CPA groups (M \pm SD)

\begin{tabular}{lllll}
\hline Variable & & High MPA Group & Low CPA Group & T \\
\hline \multirow{3}{*}{ Social Support } & Objective support & $8.49 \pm 2.11$ & $8.48 \pm 1.88$ & 0.96 \\
& subjective support & $19.38 \pm 3.64$ & $20.21 \pm 3.84$ & $0.10^{*}$ \\
& Support utilization & $7.52 \pm 1.54$ & $7.49 \pm 1.71$ & 0.89 \\
& Total social support score & $35.38 \pm 5.52$ & $36.17 \pm 5.74$ & $0.30^{*}$ \\
\hline \multirow{3}{*}{ Security } & Interpersonal security & $25.52 \pm 5.17$ & $28.51 \pm 5.34$ & $0.00^{*}$ \\
& Sense of control & $23.49 \pm 5.46$ & $27.46 \pm 5.61$ & $0.00^{*}$ \\
& Total Security Score & $49.01 \pm 9.91$ & $55.97 \pm 10.37$ & $0.00^{*}$ \\
\hline
\end{tabular}

$* \mathrm{P}<0.05, * * \mathrm{P}<0.01, \quad * * * \mathrm{P}<0.001$

\subsection{Relationship Between College Students' Mobile Phone Dependence and Social Support and Sense of Security}

Correlation analysis results showed that: (1) there was a significant negative correlation between mobile phone dependence and social support and sense of security; There was also a negative correlation between other factors that support mobile phone dependence, and the relationship is significant on social comfort; (3) There was a significant negative correlation between each factor of security and mobile phone dependence.

Table 3. The Regression Analysis of the CPA, the Social Support and the Security

\begin{tabular}{|c|c|c|c|c|c|c|c|}
\hline Variable & 1 & 2 & 3 & 4 & 5 & 6 & 7 \\
\hline Withdrawal response1 & 1 & & & & & & \\
\hline Salient Behviour & $0.67 * *$ & 1 & & & & & \\
\hline Social Comfort3 & $0.60 * *$ & $0.50 * *$ & 1 & & & & \\
\hline Mind Change4 & $0.55^{* *}$ & $0.57 * *$ & $0.44 * *$ & 1 & & & \\
\hline CPA5 & $0.90 * *$ & $0.85^{* *}$ & $0.76^{* *}$ & $0.75^{* *}$ & 1 & & \\
\hline Social Comfort6 & -0.02 & 0.001 & $-0.18 * *$ & -0.006 & $-0.06^{* *}$ & 1 & \\
\hline Security 7 & $-0.19 * *$ & $-0.20 * *$ & $-0.29 * *$ & $-0.15^{* *}$ & $-0.25^{* *}$ & $0.32 * *$ & 1 \\
\hline M & 19.11 & 10.73 & 8.27 & 8.14 & 46.25 & 35.60 & 52.37 \\
\hline SD & 4.43 & 3.25 & 2.62 & 2.35 & 10.48 & 5.47 & 9.75 \\
\hline
\end{tabular}

$* \mathrm{P}<0.05, * * \mathrm{P}<0.01, * * * \mathrm{P}<0.001$.

\subsection{Predictive Analysis of College Students' Social Support and Sense of Security on Mobile Phone Dependence}

In order to explore the predictive effect of college students' social support and sense of security on mobile phone dependence, social support and sense of security were used as predictive variables, and mobile phone dependence was used as the dependent variable. Multiple regression analysis was performed. The results showed that: (1) both social support and sense of security had a significant negative predictive effect on the total score of mobile phone dependence; (2) except for emotion changes, social support can significantly predict other sub dimensions of mobile phone dependence (3) The sense of security had a significant negative predictive effect on each factor of mobile phone dependence. 
Table 4. Regression analyses of mobile phone dependence, social support, and security

\begin{tabular}{llllll}
\hline $\begin{array}{l}\text { independent } \\
\text { variable }\end{array}$ & dependent variable & standardized regression coefficient $(\boldsymbol{\beta})$ & $\boldsymbol{t}$ & $\boldsymbol{R}^{2}$ & $\boldsymbol{F}$ \\
\hline Social Support & withdrawal symptoms & -0.02 & $-0.47^{* * *}$ & 0.001 & $0.22^{* * *}$ \\
Security & & -0.09 & $-3.79^{* * *}$ & 0.04 & $14.36^{* * *}$ \\
Social Support & Salient Behavior & 0.00 & 0.02 & 0.00 & 0.00 \\
Security & & -0.67 & $-4.10^{* * *}$ & 0.04 & $16.80^{* * *}$ \\
Social Support & Social Comfort & -0.09 & $-3.70^{* * *}$ & 0.03 & $13.71^{* * *}$ \\
Security & & -0.08 & $-6.03^{* * *}$ & 0.08 & $36.37^{* * *}$ \\
Social Support & Mood Change & -0.002 & -0.12 & 0.00 & 0.01 \\
Security & & -0.04 & $-2.93^{*}$ & 0.02 & $8.59^{*}$ \\
Social Support & MPA & -0.11 & $-1.13^{*}$ & 0.003 & 1.28 \\
Security & & -0.27 & $-5.07^{* * *}$ & 0.06 & $25.70^{* * *}$ \\
\hline
\end{tabular}

* $\mathrm{P}<0.05,{ }^{* *} \mathrm{P}<0.01, * * * \mathrm{P}<0.001$.

\subsection{Analysis of the Mediating Effect of Security on Social Support and Mobile Phone Dependence}

The mediation effect test was used to examine the mediation effect of security on social support and mobile phone dependence. The results showed that when the sense of security was used as an intermediary variable, the absolute value of the path coefficient (direct benefit c ') of social support and mobile phone dependence decreased. It can be considered that the sense of security completely mediated the role of social support and mobile phone dependence.

\section{Discussion}

\subsection{Mobile Phone of College Students is Common and Serious}

The results showed that there were no gender, grade, love, one child differences of mobile phone in college students. It can be seen that mobile phone dependence has become a common phenomenon. Mobile phone is gradually becoming the most important information exchange tool for people. In addition to the most basic functions such as call and SMS, the chat platforms, game apps and shopping is more popular. Many new functions of mobile phones are very popular, especially for young people. Mollie phone can meet most of the daily needs of people, and at the same time gradually deepen people's dependence on mobile phones.

From a gender perspective, compared with men, women used mobile apps more for consumption and shopping. In some aspects the gender difference was gradually decreasing. People will no longer think that shopping is exclusively for women and games are designed for men. The purpose of using mobile phone for men and women tend to be the same, so the difference is not significant. There was no grade difference of mobile phone addiction in college students. Sophomores and sophomores were more obsessed with fresh campus and social activities, while seniors and graduate students were more focused on work practice or academic research. This study found there was no love difference of mobile phone addiction. It is not consistent with general hypothesis. General hypothesis thinks people in love will communicate more frequently and more often use mobile phone communication, so their mobile phone addiction are more serious. Jin, Wen and Jiang (2015) thought the people in love have more mutual support and they need fewer external social than those who are not in a relationship, so those who were not in a relationship were evenly assigned a higher social comfort than those who were in a relationship.

\subsection{The Relationship Among College Students' Mobile Phone Dependence, Social Support and Sense of Security Was Significant}

The results showed that there was a negative correlation between mobile phone dependence and social support and security, which was consistent with the hypothesis of this study. Some college students were addicted to mobile phone because of insufficient social support. The interpersonal relationships of college students were relatively simple, and the main sources of social support came from family and friends. College students were in a transitional period from students to professional man. A considerable number of parents will control the financial supply to college students. At the same time, college students have a distinctive personality, and there are differences in concepts between parents and college students. At this stage, sometimes there will be conflicts between college students and their families. These conflicts will reduce students' acceptance of social support from 
family members. In addition most of college students' friends are unable to provide practical financial support and mental support. When social support is insufficient, college students will choose to use the Internet platform to vent their emotions and seek external social support. Mobile phones have become a most convenient tool, so people with lower social support have more serious mobile phone addiction.

The self-awareness of college students has not yet fully formed. Most college students do not have a clear understanding of themselves. When they leave the campus environment, they experience many setbacks, their self-confidence decreases, and they gradually build up defenses and establish self-defense against outside society. College students are accustomed to socializing through mobile phone platforms such as WeChat and QQ. Using mobile phones for communication avoids the visual impact of face-to-face social interaction and the immediacy of communication, which is a way for mobile phone users to feel more secure. Therefore, people with a lower sense of security are more dependent on the use of mobile phones for socializing.

\subsection{The Mediating Effect of Security on Social Support and Mobile Phone Dependence}

This work suggested that the negative predictive effect of social support and security on mobile phone dependence, so improving the social support and security situation of college students could effectively change the situation of college students' mobile phone dependence. The mediating effect further showed that the sense of security has a complete intermediary effect between social support and mobile phone dependence. Increasing the social support of college students can significantly improve their sense of security, and improving their sense of security can significantly reduce the degree of mobile phone dependence. In order to build a strong sense of security the college students should enhance self-worth and self-confidence. At the same time, college students should also communicate more with their parents, combine their ideas with their parents' suggestions and improve the acceptance of external support. The increase in social support, especially the support of parents and families, will bring a great sense of security to college students (Li, 2013; Wei, 2012). The improvement of the sense of security will promote the self-confidence of college students and urge college students to join more social practices and collective activities. More actual social interaction could reduce the intensity and frequency of mobile phone use and effectively reduce college students' mobile phones.

\section{Conclusions}

In order to further study the current situation and relationship of college students 'mobile phone dependence, social support and sense of security, a questionnaire survey was performed on 409 college students using the college students' mobile phone addiction tendency scale, social support assessment scale and sense of safety scale. The results showed that: (1) $40.24 \%$ of college students are moderate of mobile phone dependence, and $5.75 \%$ of college students had serious problem of mobile phone dependence; the social support and sense of security of college students were at the upper-middle level; there is no gender difference, no one child difference and no love difference of mobile phone dependence in college students; there is grade difference of mood change; (2) the social support and sense of security of people with high phone dependence are significantly lower than those with low phones Dependence; (3) Social dependence and sense of security have significant negative correlations and negative predictive effects on mobile phone dependence; (4) Security sense has a full mediating role between social support and mobile phone dependence, thus enhancing social support and the sense of security of college students can effectively improve their mobile phone dependence.

This research had some innovations. It studied the relationship between mobile phone dependence and sense of security of undergraduates who had less research at present, which added materials and theoretical basis for the research of mobile phone dependence among college students. However, there are still inadequacies in this study. First, due to the uncertainty of the subjects of the online questionnaire, the proportion of men and women in the survey was not balanced. Secondly, the seniors were mainly senior students, the distribution was not balanced, and they were not in a relationship. In this study, the formation of college students 'sense of security and the sources of social support can be further explored in order to make more specific suggestions for alleviating college students' dependence on mobile phones.

\section{References}

Chóliz, M., Pinto, L., Phansalkar, S. S., Corr, E., Mujjahid, A., Flores, C., \& Barrientos, P. E. (2016). Development of a brief multicultural version of the test of mobile phone dependence (TMDbrief) questionnaire. Frontiers in psychology, 7, 650. https://doi.org/10.3389/fpsyg.2016.00650

Cong, Z., \& An, L. (2004). Preliminary Compilation of Safety Sense Scale and Reliability and Validity Tests. Chinese Mental Health Journal, 2, 97-99.

Ding, W. (2014). Study on the Relationship between Personality Traits, Social Support and Mobile Phone 
Dependence of College Students. Master's thesis, Guangxi Normal University.

Guo, M., Zhang, P., \& Zhang, Y. (2014). College freshmen adaptation and sense of security, life events, social support. Chinese Journal of Health Psychology, 4, 584-585. https://doi.org/10.13342/j.cnki.cjhp.2014.04.042

LUO, Y. H., HUANG, Y. P., \& YOU, M. H. (2011). Mobile Phone's Influence on College Students and Its Countermeasures [J]. Journal of Chongqing University of Posts and Telecommunications (Social Science Edition), 2. https://doi.org/10.16309/j.cnki.1007-1776.2015.09.021

Jiang, Y., \& Bai, X. (2014). The Relationship between College Students' Mobile Internet Dependence and Loneliness: The Mediating Role of Network Social Support. China Special Education, 1, 41-47.

Jin, R., Wen, X., \& Jiang, Y. (2015). The Relationship between College Students' Social Support and Mobile Phone Dependence: The Mediating Role of Social Adaptation. Journal of Guangzhou University (Social Science Edition), 10, 59-64.

Ruthig, J. C., Haynes, T. L., Stupnisky, R. H., \& Perry, R. P. (2009). Perceived academic control: Mediating the effects of optimism and social support on college students' psychological health. Social psychology of education, 12(2), 233-249. https://doi.org/10.1007/s11218-008-9079-6

Li, J. (2013). Research on the Relationship between College Students' Sense of Security, Self-acceptance and Social Support. Journal of Shanxi Datong University (Natural Science Edition), 6, 66-70.

Li, Y., \& Wang, Y. (2008). Correlation Analysis of Understanding Social Support and Security and College Students' Suicide Attitudes. Chinese Journal of School Health, 2, 141-143.

Liu, P. (2014). A Study on the Relationship between Mobile Phone Dependence, Social Support and Personality Traits of College Students (Master's thesis, Central China Normal University).

Liu, W., \& Cai, T. (2015). The relationship between social support and college students' mobile phone dependence tendency: the mediating role of loneliness. Chinese Journal of Clinical Psychology, 5, 926-928. https://doi.org/10.16128/j.cnki.1005-3611.2015.05.039

Meng, H., Wang, Y., \& Feng, C. (2007). Analysis of factors related to psychological safety of college students. Chinese Journal of Tissue Engineering Research and Clinical Rehabilitation, 39, 7880-7883.

Statistical Bulletin of the Communications Operations Industry in 2018. Retrieved from http://www.miit.gov.cn/n1146285/n1146352/n3054355/n3057511/n3057518/c6618525/content.html

Wang, F. (2016). Mobile phone dependence of college students and its relationship with loneliness and social support. Journal of Jiamusi Vocational College, 2, 444-445.

Wang, Y., Zou, Z., Song, H., Xu, X., Wang, H., d'Oleire Uquillas, F., \& Huang, X. (2016). Altered gray matter volume and white matter integrity in college students with mobile phone dependence. Frontiers in psychology, 7, 597. https://doi.org/10.3389/fpsyg.2016.00597

Wei, Y. (2012). Research on the Relationship between College Students' Social Support and Mobile Phone Dependence. Journal of Jilin Business and Technology College, 4, 91-93.

Xiao, S. (1994). Theoretical basis and research application of the Social Support Rating Scale. Journal of Clinical Psychiatry, 2, 98-100.

Xiong, J., Zhou, Z. K., Chen, W., You, Z. Q., \& Zhai, Z. Y. (2012). Development of the mobile phone addiction tendency scale for college students. Chin. Ment. Health J, 26, 222-225.

Zhang, F., Liu, Z., \& Fan, C. (2014). Impact of Internet Social Support and Internet Security on College Students' Subjective Well-Being. Education Review, 2, 52-54.

Zhao, W. (2016). The relationship between mobile phone dependence of young students and interpersonal trust on the Internet: the mediating role of security. Journal of Northern University for Nationalities (Philosophy and Social Sciences), 6, 102-105.

\section{Copyrights}

Copyright for this article is retained by the author(s), with first publication rights granted to the journal.

This is an open-access article distributed under the terms and conditions of the Creative Commons Attribution license (http://creativecommons.org/licenses/by/4.0/). 